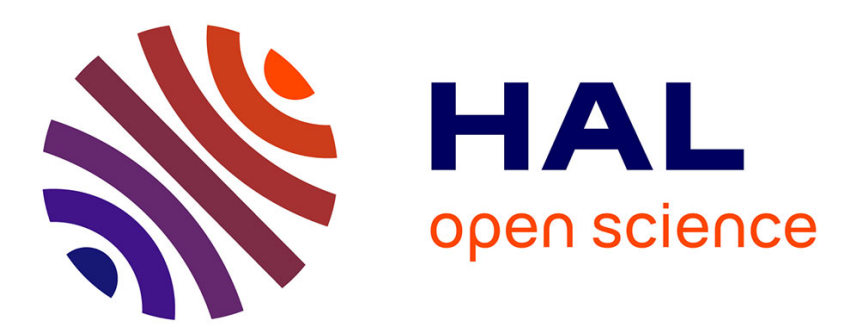

\title{
The method of fundamental solutions for acoustic wave scattering by a single and a periodic array of poroelastic scatterers
}

\author{
Benoit Nennig, Emmanuel Perrey-Debain, Jean-Daniel Chazot
}

\section{- To cite this version:}

Benoit Nennig, Emmanuel Perrey-Debain, Jean-Daniel Chazot. The method of fundamental solutions for acoustic wave scattering by a single and a periodic array of poroelastic scatterers. Engineering Analysis with Boundary Elements, 2011, 35 (8), pp.1019-1028. 10.1016/j.enganabound.2011.03.007 . hal-00694764

\section{HAL Id: hal-00694764 \\ https://hal.science/hal-00694764}

Submitted on 6 May 2012

HAL is a multi-disciplinary open access archive for the deposit and dissemination of scientific research documents, whether they are published or not. The documents may come from teaching and research institutions in France or abroad, or from public or private research centers.
L'archive ouverte pluridisciplinaire HAL, est destinée au dépôt et à la diffusion de documents scientifiques de niveau recherche, publiés ou non, émanant des établissements d'enseignement et de recherche français ou étrangers, des laboratoires publics ou privés. 


\title{
The method of fundamental solutions for acoustic wave scattering by a single and a periodic array of poroelastic scatterers
}

\author{
B. Nennig, ${ }^{\mathrm{a}, *}$ E. Perrey-Debain ${ }^{\mathrm{a}}$, J.-D. Chazot ${ }^{\mathrm{a}}$ \\ ${ }^{a}$ Université de Technologie de Compiègne, Laboratoire Roberval UMR 6253, BP 20529,60205 Compiègne CEDEX, France
}

\begin{abstract}
The Method of Fundamental Solutions (MFS) is now a well-established technique that has proved to be reliable for a specific range of wave problems such as the scattering of acoustic and elastic waves by obstacles and inclusions of regular shapes. The goal of this study is to show that the technique can be extended to solve transmission problems whereby an incident acoustic pressure wave impinges on a poroelastic material of finite dimension. For homogeneous and isotropic materials, the wave equations for the fluid phase and solid phase displacements can be decoupled thanks to the Helmholtz decomposition. This allows for a simple and systematic way to construct fundamental solutions for describing the wave displacement field in the material. The efficiency of the technique relies on choosing an appropriate set of fundamental solutions as well as properly imposing the transmission conditions at the air-porous interface. In this paper, we address this issue showing results involving bidimensional scatterers of various shapes. In particular, it is shown that reliable error indicators can be used to assess the quality of the results. Comparisons with results computed using a mixed pressure-displacement finite element formulation illustrate the great advantages of the MFS both in terms of computational resources and mesh preparation. The extension of the method for dealing with the scattering by an infinite array of periodic scatterers is also presented.
\end{abstract}

Keywords: Method of fundamental solutions, Biot's equations, poroelastic, porous material, scattering

\section{Introduction}

Poroelastic materials are often used for their good sound absorbing capabilities in the middle and high frequency range. Typical applications can be found in the context of the transport noise reduction or to enhance the quality of room acoustics. The description of wave propagation in porous media is not limited to audible acoustics as Biot's model [8] was originally developed for geological applications. Because of their inherent diphasic features and the strong contrasts that may exist between the solid and the fluid phases, wave propagation modeling remains a difficult task often leading to heavy computational costs. In the context of the Finite Element Method (FEM), some developments have been proposed using Lagrange or hierarchical finite elements $[5,18,29]$. Because of the scale disparity, the so-called poroelastic elements have a slower convergence rate than purely elastic or fluid elements [29]. To make matters worse, Biot's equations are frequency dependent and large FEM system matrices have to be recalculated for each frequency. For homogeneous and isotropic materials, the Boundary Element Method (BEM) offers an alternative [31]. The method has the advantage of reducing the entire problem to one with only unknowns on the

* Corresponding author. Present address : LAUM UMR CNRS 6613, université du Maine, Avenue Olivier Messiaen, 72085 Le Mans CEDEX 9, France, benoit.nennig@univ-lemans.fr.

Email address: benoit.nennig@utc.fr (B. Nennig) boundaries. However, the system matrix is full and there is still the need to discretize the boundary surface as well as performing regular and singular integrations over each boundary element.

In the past decade, several researchers have focused their work on meshless methods in order to avoid the time-consuming mesh generation process for complicated geometries. In this regard, the Method of Fundamental Solution (MFS) has been shown to be efficient for solving a large variety of physical problems as long as a fundamental solution of the underlying differential equation(s) is known. In particular, the MFS is suitable for scattering problems by choosing appropriate fundamental solutions satisfying the radiation condition at infinity. The method shares the same advantages as the BEM over domain discretization methods because there is no need to create a mesh over the entire domain. Furthermore, as no integration is needed, some numerical difficulties encountered with the BEM are avoided. For comprehensive reviews on applications of the MFS for scattering and radiation problems one can refer to Fairweather et al. $[13,14]$.

In this work, we are interested in applying as well as assessing the MFS for the numerical simulation of a bidimensional incident acoustic wave scattered by a poroelastic material. To the authors' knowledge, such problems have never been addressed using the MFS and although analytical solutions are available for canonical geometries 


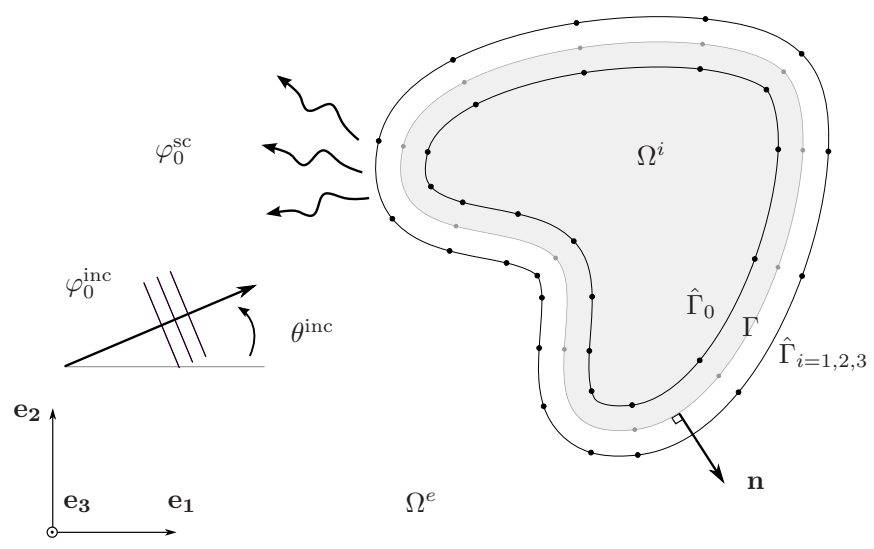

Figure 1: Scattering geometry.

such as cylindrical and spherical scatterers [15, 16], there is a need for fast and accurate methods taking into account scatterers of arbitrary shape. The applications we have in mind range from multiple scattering, the modeling of double porosity materials $[6,26]$ to shape foam optimization modeling [19, 30].

In a preliminary note presented by the authors [25], the application of the MFS to such problems was briefly presented. In this work, all the details of the implementation of the method and the investigation of its accuracy and numerical characteristics, including the condition number of the resulting matrices are given. The present paper is organized as follows. After presenting the MFS formulation in Section 2, the method's performance is measured with respect to an academic problem, the circular-shaped scatterer, for which analytical solutions are available. In particular, it is shown that reliable error indicators can be used to assess the quality of the results. In Section 4, various numerical examples of increasing difficulty are presented with a comparison to FEM results. The last section shows applications concerning the scattering of an incident plane wave by an infinite periodic array of porous structures.

\section{Formulation of the method}

Consider a time-harmonic acoustic plane wave $\varphi_{0}^{\text {inc }}=$ $A^{\text {inc }} \exp \mathrm{i} k_{0} \zeta \cdot \mathbf{x}$ (with the convention $\mathrm{e}^{-\mathrm{i} \omega t}$ ) in an unbounded exterior domain $\Omega^{e}$ incident upon one (or more) poroelastic acoustic foam(s) denoted by $\Omega^{i}$ with boundary $\Gamma$ as shown in Fig. 1. We denote by $\mathbf{n}$, the unit outward normal vector to $\Omega^{i}$. In the surrounding acoustic domain $\Omega^{e}$, the fluid is inviscid and the acoustic displacement potential $\varphi_{0}$ obeys the wave equation

$$
\Delta \varphi_{0}+k_{0}^{2} \varphi_{0}=0
$$

Here, $k_{0}=\omega / c_{0}$ is the classical wavenumber defined as the ratio of the angular frequency $\omega$ and the sound speed $c_{0}$. To express the transmission conditions at the interface $\Gamma$, it is convenient to introduce the particle displacement perturbation $\mathbf{w}=\nabla \varphi_{0}$. With this definition, the acoustic pressure is obtained from the linearized momentum equation as $p=\rho_{0} \omega^{2} \varphi_{0}$. In (1), it is natural to split the potential into an incident and a scattered part as $\varphi_{0}=\varphi_{0}^{\text {inc }}+\varphi_{0}^{\text {sc }}$ (and similarly for the pressure and displacement). Here we require the scattered field to satisfy the usual Sommerfeld radiation condition at infinity. In the poroelastic medium the acoustic waves propagation is described by Biot's model [8]. This latter is based on the superposition of a fluid phase and a solid phase which are coupled together and respectively described by the fluid phase displacement $\mathbf{U}$ and the solid phase displacement $\mathbf{u}$. For the time-harmonic representation, we have the following coupled system [8]

$$
\begin{aligned}
& \nabla \cdot \boldsymbol{\sigma}^{s}+\omega^{2}\left(\rho_{11} \mathbf{u}+\rho_{12} \mathbf{U}\right)=0, \\
& \nabla \cdot \boldsymbol{\sigma}^{f}+\omega^{2}\left(\rho_{12} \mathbf{u}+\rho_{22} \mathbf{U}\right)=0 .
\end{aligned}
$$

The solid and fluid phase stress tensors are given by

$$
\begin{aligned}
& \boldsymbol{\sigma}^{s}=(A \nabla \cdot \mathbf{u}+Q \nabla \cdot \mathbf{U}) \mathbf{I}+2 N \boldsymbol{\varepsilon}^{s}, \\
& \boldsymbol{\sigma}^{f}=(Q \nabla \cdot \mathbf{u}+R \nabla \cdot \mathbf{U}) \mathbf{I},
\end{aligned}
$$

where $\varepsilon^{s}=1 / 2\left(\nabla \mathbf{u}+(\nabla \mathbf{u})^{\mathrm{t}}\right)$ is the usual strain tensor and $\mathbf{I}$ is the identity matrix. The total stress tensor $\boldsymbol{\sigma}^{t}$ is, by definition, the sum of $\boldsymbol{\sigma}^{f}$ and $\boldsymbol{\sigma}^{s}$. Biot's coefficients $A, N, Q, R$ are related to the material properties by the Allard-Johnson model. Their expressions can be found in the literature or in the reference textbook [2] as well as the other quantities introduced in this section. These quantities are all complex and frequency-dependent, $A$ and $N$ correspond to the Lamé coefficients, $R$ is the effective bulk modulus of the fluid phase and $Q$ indicates the coupling of the two phases volumic dilatation. The imaginary parts of $A$ and $N$ include the structural damping and, in $Q$ and $R$ these parts include the thermal dissipation. The imaginary parts of the effective density coefficients $\rho_{11}, \rho_{22}$ and $\rho_{12}$ take into account viscous damping. The complete solution to the problem is found after applying the classical air-porous transmission conditions $[11,22]$ on the interface $\Gamma$, i.e.

$$
\begin{aligned}
p_{p}-p^{\mathrm{sc}} & =p^{\mathrm{inc}}, \\
\phi \mathbf{U} \cdot \mathbf{n}+(1-\phi) \mathbf{u} \cdot \mathbf{n}-\mathbf{w}^{\mathrm{sc}} \cdot \mathbf{n} & =\mathbf{w}^{\mathrm{inc}} \cdot \mathbf{n}, \\
\boldsymbol{\sigma}^{t} \mathbf{n}+p^{\mathrm{sc}} \mathbf{n} & =-p^{\mathrm{inc}} \mathbf{n} .
\end{aligned}
$$

Here $\phi$ is the porosity and the pore pressure $p_{p}$ is obtained from the fluid phase tensor as $-\mathbf{I} \phi p_{p}=\boldsymbol{\sigma}^{f}$.

For homogeneous and isotropic materials, the wave equation for the fluid phase and solid phase displacements can be decoupled thanks to the Helmholtz decomposition. Both solid and fluid displacement fields are then written as

$$
\mathbf{u}=\nabla \varphi+\nabla \wedge\left(\varphi_{3} \cdot \mathbf{e}_{3}\right) \text { and } \mathbf{U}=\nabla \chi+\nabla \wedge\left(\Theta \cdot \mathbf{e}_{3}\right) .
$$

After decoupling the equations, we have [2]: $\varphi=\varphi_{1}+\varphi_{2}$ and $\chi=\mu_{1} \varphi_{1}+\mu_{2} \varphi_{2}$ where

$$
\mu_{i}=\frac{P k_{i}^{2}-\omega^{2} \rho_{11}}{\omega^{2} \rho_{12}-Q k_{i}^{2}}, \quad i=1,2,
$$


are the wave amplitude ratios between the two phases in the porous material (here, $P=A+2 N$ ). Similarly, the potential $\Theta$ is simply obtained as $\Theta=\mu_{3} \varphi_{3}$ with $\mu_{3}=$ $\rho_{12} / \rho_{22}$. Under this form, each potential $\varphi_{i}(i=1,2,3)$ satisfies the Helmholtz equation

$$
\Delta \varphi_{i}+k_{i}^{2} \varphi_{i}=0
$$

and the associated complex wavenumbers are

$$
\begin{aligned}
k_{1}^{2} & =\frac{\omega^{2}}{2\left(P R-Q^{2}\right)}\left(P \rho_{22}+R \rho_{11}-2 Q \rho_{12}+\sqrt{D}\right), \\
k_{2}^{2} & =\frac{\omega^{2}}{2\left(P R-Q^{2}\right)}\left(P \rho_{22}+R \rho_{11}-2 Q \rho_{12}-\sqrt{D}\right), \\
k_{3}^{2} & =\frac{\omega^{2}}{N}\left(\frac{\rho_{11} \rho_{22}-\rho_{12}^{2}}{\rho_{22}}\right) .
\end{aligned}
$$

Here, $D$ stands for the discriminant of a quadratic equation and $D=\left(P \rho_{22}+R \rho_{11}-2 Q \rho_{12}\right)^{2}-4\left(P R-Q^{2}\right)\left(\rho_{11} \rho_{22}-\right.$ $\left.\rho_{12}^{2}\right)$. Physically, there are two compressional waves associated with $\varphi_{1}, \varphi_{2}$ and one rotational (shear) wave associated with $\varphi_{3}$. They all propagate in the two phases and their relative contributions are given by the coefficients $\mu_{i}$. If such a decomposition holds in elastodynamics, the coexistence of two phases in the poroelastic media adds another fluid-borne compressional wave which is not present is elastic solids.

The MFS implementation starts by choosing an appropriate set of fundamental solutions for both propagative domains $\Omega^{e}$ and $\Omega^{i}$. In the acoustic domain, a natural choice is to choose these solutions using the well-known free field Green's function, i.e. $G_{0}(\mathbf{x}, \mathbf{y})=\mathrm{i} / 4 H_{0}\left(k_{0}|\mathbf{x}-\mathbf{y}|\right)$. So, we seek the scattered field as a distribution of monopoles

$$
p^{\mathrm{sc}}(\mathbf{x})=\rho_{0} \omega^{2} \sum_{q_{0}=1}^{Q_{0}} A_{q_{0}}^{0} G_{0}\left(\mathbf{x}, \mathbf{y}_{q_{0}}^{0}\right)
$$

where the source points $\mathbf{y}_{q_{0}}^{0}$ are chosen to be located on a curve $\hat{\Gamma}_{0}$ in the interior domain $\Omega^{i}$. The displacement vector is obtained by simply taking the gradient of (12). To simulate the wave field in the foam, a possible choice is to use fundamental solutions for poroelastic media for which an explicit form can be found, for instance, in [9]. An easier option is to construct these solutions by simply expanding each potential in the form

$$
\varphi_{i}(\mathbf{x})=\sum_{q_{i}=1}^{Q_{i}} A_{q_{i}}^{i} G_{i}\left(\mathbf{x}, \mathbf{y}_{q_{i}}^{i}\right), \quad \text { for } i=1,2,3,
$$

where $G_{i}(\mathbf{x}, \mathbf{y})=\mathrm{i} / 4 H_{0}\left(k_{i}|\mathbf{x}-\mathbf{y}|\right)$ is the fundamental solution of the Helmholtz equation. Here, $Q_{i}$ is the number of source points $\mathbf{y}_{q_{i}}^{i}$ associated with the $i^{\text {th }}$ potential and the $A_{q_{i}}^{i}$ 's are unknown amplitudes. These points are located on a fictitious boundary $\hat{\Gamma}_{i}$ in the exterior domain $\Omega^{e}$ as shown in Fig. 1. The influence of the location of the source points on the quality of the solution will be discussed in the next section. Now, using these expansions in
(6), we find the expression for the different physical quantities involved:

$$
\begin{aligned}
\phi p_{p}(\mathbf{x}) & =\sum_{i=1}^{2} k_{i}^{2}\left(Q+\mu_{i} R\right) \sum_{q_{i}=1}^{Q_{i}} A_{q_{i}}^{i} G_{i}\left(\mathbf{x}, \mathbf{y}_{q_{i}}^{i}\right) \\
\mathbf{u}(\mathbf{x}) & =\sum_{i=1}^{2} \sum_{q_{i}=1}^{Q_{i}} A_{q_{i}}^{i} \nabla G_{i}\left(\mathbf{x}, \mathbf{y}_{q_{i}}^{i}\right)+\sum_{q_{3}=1}^{Q_{3}} A_{q_{3}}^{3} \nabla_{\perp} G_{3}\left(\mathbf{x}, \mathbf{y}_{q_{3}}^{3}\right) \\
\mathbf{U}(\mathbf{x}) & =\sum_{i=1}^{2} \sum_{q_{i}=1}^{Q_{i}} A_{q_{i}}^{i} \mu_{i} \nabla G_{i}\left(\mathbf{x}, \mathbf{y}_{q_{i}}^{i}\right) \\
& +\sum_{q_{3}=1}^{Q_{3}} A_{q_{3}}^{3} \mu_{3} \nabla_{\perp} G_{3}\left(\mathbf{x}, \mathbf{y}_{q_{3}}^{3}\right)
\end{aligned}
$$

where $\nabla_{\perp} \equiv\left(\partial_{x_{2}},-\partial_{x_{1}}\right)^{\mathrm{t}}$ stands for the orthogonal gradient operator. From these expressions and using (4), we finally obtain the normal total stress tensor $\boldsymbol{\sigma}^{t}$. The explicit forms for the tensor coefficients are too cumbersome to be inserted in this paper. Note that other type of solutions could have been considered (Green's functions and/or their derivatives or plane waves for instance) and the present choice was mainly motivated by simplicity. Furthermore, it has the advantage of allowing us to specify independently the number of source points $Q_{i}$ for each kind of wave, which is not the case when using Green's functions. In this respect, our method is not strictly speaking an MFS since it does not rely on Biot's equations fundamental tensor.

Now, given a set of $N_{\text {col }}$ collocation points $\mathbf{x}_{l}(l=$ $1, \ldots, N_{\text {col }}$ ) on $\Gamma$, substituting (14) in the transmission conditions (5) at each collocation point yields three linear systems of the form

$$
\mathbf{M}_{\alpha} \mathbf{A}=\mathbf{F}_{\alpha}^{\mathrm{inc}}, \quad \alpha=\mathrm{a}, \mathrm{b}, \mathrm{c} .
$$

Here, the subscript $\alpha$ refers to the type of condition involved (there is one system for each condition (5a), (5b), $(5 c))$. The right hand side vector $\mathbf{F}_{\alpha}^{\text {inc }}$ stems from the incident wave (pressure and displacement). The unknown vector A contains the amplitudes of all sources. Since the acoustic displacement is expected to behave like $|\mathbf{w}| \sim$ $k_{0} \phi_{0}$, it is judicious to rescale the kinematic conditions (5b) by multiplying the associated lines by $\rho_{0} c_{0} \omega$. This yields the matrix system

$$
\mathbf{M A}=\mathbf{F}^{\text {inc }} .
$$

In this work, early results showed that it is preferable for reasons of stability to consider more collocation points than the number of unknowns $N_{\text {dof }}=\sum_{i=0}^{3} Q_{i}$. To be more specific, it was found that taking $N_{\text {col }}=2 N_{\text {dof }}$ guarantees that results have converged, i.e. the numerical solution becomes insensitive to the number of collocation points, as discussed in [3]. All calculations were performed using this ratio. Note that when dealing with 'nice' shaped 
scatterers (the circular scatterer for instance), the MFS has been observed to perform slightly better with interpolation schemes (i.e. $N_{\text {col }}=N_{\text {dof }}$ ) than least-squares schemes $\left(N_{\text {col }}>N_{\text {dof }}\right)$. However, for the sake of robustness and generality the second approach was preferred. After multiplying the system (16) by the Hermitian transpose $\mathbf{M}^{\dagger}$ we finally get the Hermitian square system

$$
\mathbf{M}^{\dagger} \mathbf{M A}=\mathbf{M}^{\dagger} \mathbf{F}^{\text {inc }}
$$

A important advantage of performing this operation is that a simple error estimator is available at very low cost. Indeed, going back to the original collocation problem (15), we can define the a posteriori algebraic error estimator for each of the conditions at the interface $(\alpha=\mathrm{a}, \mathrm{b}, \mathrm{c})$. We thus define

$$
\mathcal{E}_{\alpha}=100 \frac{\left\|\mathbf{M}_{\alpha} \mathbf{A}-\mathbf{F}_{\alpha}^{\mathrm{inc}}\right\|_{2}}{\left\|\mathbf{F}_{\alpha}^{\mathrm{inc}}\right\|_{2}},
$$

where $\mathbf{A}$ is solution of (17). The numerical evaluation of (18) is computationally cheap since the matrices $\mathbf{M}_{\alpha}$ have already been calculated. Of course, taking $N_{\text {col }}=N_{\text {dof }}$ will automatically produce errors with zero value (except from when interpolation matrices are very ill-conditioned). By increasing the number of collocation points, errors will grow until it stabilises.

As for the choice of positions of the source points, we follow Alves [3] and take these points along the discrete normal direction, so we put $\mathbf{y}_{q_{i}}^{i}=\hat{\mathbf{y}}_{q_{i}}^{i}+s^{i} \tilde{\mathbf{n}}_{q_{i}}^{i}$ where the points $\hat{\mathbf{y}}_{q_{i}}^{i}\left(q_{i}=1, \ldots, Q_{i}\right.$ and $\left.i=0, \ldots, 3\right)$ are distributed on $\Gamma$ and $\tilde{\mathbf{n}}_{q_{i}}^{i}$ is the approximate normal vector defined by

$$
\tilde{\mathbf{n}}_{q_{i}}^{i}=\frac{\left(\hat{\mathbf{y}}_{q_{i}+1}^{i}-\hat{\mathbf{y}}_{q_{i}-1}^{i}\right)^{\perp}}{\max _{q_{i}}\left|\hat{\mathbf{y}}_{q_{i}+1}^{i}-\hat{\mathbf{y}}_{q_{i}-1}^{i}\right|}
$$

Here, the symbol $\perp$ signifies that we take the orthogonal vector pointing outward. The normalization is chosen here for convenience to ensure that the normal vector amplitudes never exceed unity and that coefficients $s^{i}$ correspond to the farthest distance from the interface $\Gamma$.

Before we end this section, we should point out that, as far as engineering applications are concerned, the physical variables of primary interest are the pressure and the normal velocity at the interface. In practical terms, the scattering characteristics of the porous obstacle are conveniently quantified by the scattering cross section (SCS) defined as the ratio between the scattered power and the incident power flux [23]

$$
\Sigma=\frac{W^{\mathrm{sc}}}{I^{\text {inc }}}=\frac{\int_{\Gamma} \mathfrak{R e}\left(p^{\mathrm{sc}} \overline{v_{n}^{\mathrm{sc}}}\right) \mathrm{d} \Gamma}{\left|A^{\text {inc }}\right|^{2} \omega^{4} \rho_{0} / c_{0}},
$$

where $v_{n}^{\mathrm{sc}}=-\mathrm{i} \omega \mathbf{w}^{\mathrm{sc}} \cdot \mathbf{n}$ is the normal acoustic velocity for the scattered field. Similarly, we can define the absorption cross section as the ratio between the total power and the incident power flux. These quantities combine the reflection, transmission and the absorption properties of the porous scatterer. By construction, the SCS is a far field estimator and numerical errors at the interface only have a mild impact on its numerical evaluation. This will be commented further when necessary.

\section{Validations for the circular-shaped scatterer}

We shall validate and assess the efficiency of the method in the specific case of the scattering of a horizontal acoustic plane wave by a circular-shaped poroelastic scatterer. In polar co-ordinates, the inner and outer wave fields can be represented by separable solutions. Each potential is given by infinite series which are well behaved allowing us to produce very accurate results without deterioration at high frequency. The derivation of the exact form for these solutions is quite lengthy and this is inserted in Appendix. For a proper assessment of the method's performance, it is convenient to define the relative error on the boundary for each physical quantity (call it $X$ ):

$$
\hat{\mathcal{E}}(X)=100 \frac{\|\mathbf{X}-\hat{\mathbf{X}}\|_{2}}{\|\hat{\mathbf{X}}\|_{2}},
$$

where $X$ represents either the acoustic pressure, the fluid phase or solid phase normal displacement or the normal stress at the interface and $\mathbf{X}$ is the associated vector containing the value of $X$ at the collocation points. Here, $\hat{\mathbf{X}}$ stands for a reference solution vector, either obtained analytically in the present case or computed numerically with another method when analytical solutions are not available. The aim of this section is to identify the effects of the main parameters of the problem, namely the locations and the number of sources. For this latter, we are still left with the problem of finding a quasi-optimal relationship between the $Q_{i}$ 's. Through intensive calculations, not presented here, we found that choosing the same number for each wave type was probably the best option offering the best trade-off between accuracy, conditioning and simplicity. This simplifies the analysis significantly as we can now put

$$
Q=Q_{i} \quad \text { for } \quad i=0, \ldots, 3,
$$

and perform the analysis with a single parameter $Q$. Similarly, we take the points $\hat{\mathbf{y}}_{q_{i}}^{i}$ all equal and put $s^{i}=s$ with $s^{0}=-s$. Note that this choice seems to be contrary to the results given in [27] where it is advocated that the best option, when using plane waves, is precisely not to take the same number of wave directions for each type of waves (that is shear and pressure wave types in the elasticity case) and this is particularly relevant when the ratio between the wavenumbers is large. The reasons for this probably lie in that, plane waves and singular sources behave differently in terms of their approximation properties and it would be interesting to explore this further via numerical experiments in the spirit of [4] for instance. 


\begin{tabular}{cccccccccc}
\hline \hline Material & $\phi$ & $\sigma\left(\mathrm{kNm}^{-4} \mathrm{~s}\right)$ & $\alpha_{\text {inf }}$ & $\Lambda(\mu \mathrm{m})$ & $\Lambda^{\prime}(\mu \mathrm{m})$ & $\rho_{1}\left(\mathrm{kgm}^{-3}\right)$ & $N(\mathrm{kPa})$ & $\nu$ & Ref. \\
\hline XFM foam & 0.98 & 13.5 & 1.7 & 80 & 160 & 30 & $200(1-0.05 \mathrm{i})$ & 0.35 & {$[11]$} \\
B wool & 0.95 & 23 & 1 & 54.1 & 162.3 & 58 & $8.5(1-0.1 \mathrm{i})$ & 0 & {$[12]$} \\
\hline \hline
\end{tabular}

Table 1: Materials properties. With the resistivity $\sigma$, the tortuosity $\alpha_{\text {inf }}$, the viscous and thermal characteristic lengths $\Lambda$ and $\Lambda^{\prime}$, the poisson coefficient $\nu$ and the effective skeleton density $\rho_{1}$. The effective skeleton density $\rho_{1}=(1-\phi) \rho_{s}$, where $\rho_{s}$ is the density of the material of the frame.

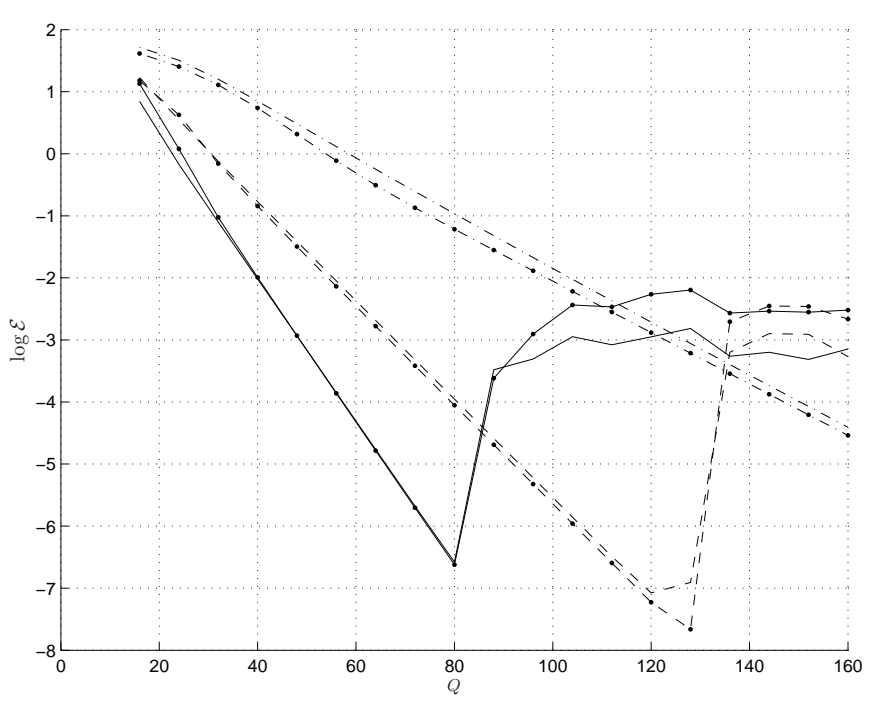

(a)

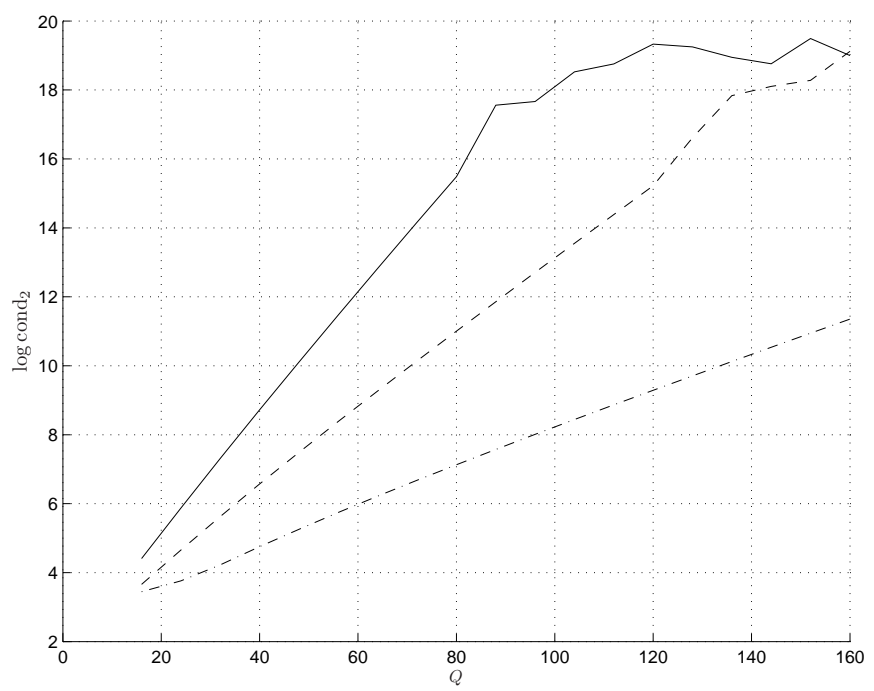

(b)

Figure 2: Effects of the sources location on the error and the conditioning $(--s=0.1 a,--s=0.2 a,-s=0.3 a)$. (a): error on the solid phase normal displacement for the XFM foam at $1500 \mathrm{~Hz}$; line with markers '.' refers to $\hat{\mathcal{E}}\left(u_{n}\right)$ error and the simple line to the a posteriori error $\mathcal{E}_{\mathrm{b}}$. (b): conditioning (2-norm) of $\mathbf{M}^{\dagger} \mathbf{M}$.
As an exemple we chose a polymer foam (XFM) commonly used in the transport industry. The material properties are reported in Table 1 . To give an idea of the complex wavenumbers for the wave potentials in the material, we find that $k_{1} \approx 17.6+1.21 \mathrm{i}, k_{2} \approx 21.4+14.1 \mathrm{i}$ and $k_{3} \approx 38.9+2.1 \mathrm{i}$ (these values correspond to the frequency $500 \mathrm{~Hz}$ ). The corresponding acoustic wavenumber is $k_{0} \approx 9.2$. On Fig. 2 (a) we show the influence of the source locations as well as the number of sources (i.e. $Q$ ) on the normal solid displacement error $u_{n}=\mathbf{u} \cdot \mathbf{n}$, i.e. $\hat{\mathcal{E}}\left(u_{n}\right)$ and the a posteriori error $\mathcal{E}_{\mathrm{b}}$. The evolution of the conditioning (in the 2-norm) of the associated system is also shown (Fig. 2 (b)). Before we comment on these results, we should point out that we chose to measure the normal displacement error rather than the pressure error for the simple reason that it was observed, that a small error on the displacement normally guarantees a smaller error on the pressure. The convergence curves together with the conditioning curves typically illustrate the MFS paradox which many authors have already observed and discussed: despite the ill-conditioning of the MFS systems, the method produces accurate results. In the present situation, it is concluded that the further away from the boundary the source points are (i.e. the larger $s$ ), the better the results as long as the conditioning of the system does not exceed a certain value above which results are likely to be corrupted by round off errors. This value, say cond $_{2} \approx 10^{16}$, is in line with standard double precision arithmetic. Note that, in our algorithm, matrices are inverted using the pinv function from MATLAB so that for very ill-conditioned matrices $\left(\operatorname{cond}_{2}>10^{16}\right)$ the SVD solver is automatically used with some thresholding in order to dampen the effects of the round-off errors. In this respect, we believe that better results could perhaps be obtained by properly filtering the small singular values. Another approach would be to apply Tikhonov regularization in order to speed up the computation. In fact, the most striking feature of these convergence curves is the almost perfect agreement between the true error $\hat{\mathcal{E}}\left(u_{n}\right)$ and its algebraic counterpart, the a posteriori error $\mathcal{E}_{\mathrm{b}}$ and through many numerical experiments, we always observed very good correlations. The mathematical reasons for this goes beyond the scope of this paper but we believe that in most cases the algebraic a posteriori errors $\mathcal{E}_{\alpha}$ should serve as reliable error indicators especially when reference solutions are not available. 


\section{Numerical examples}

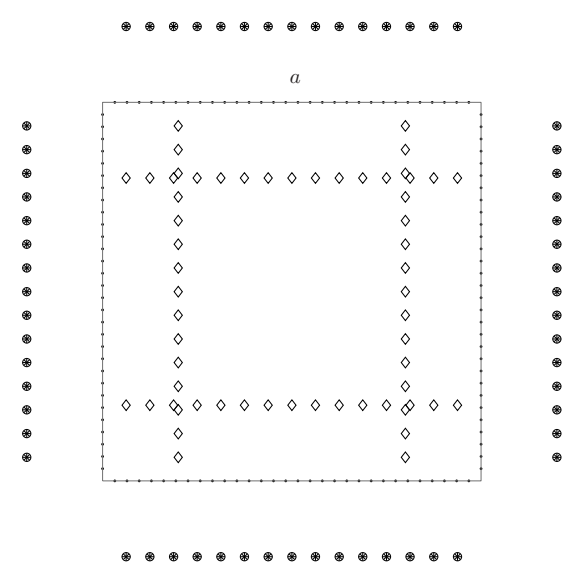

Figure 3: Square-shaped scatterer. Here symbols,$+ \times, \circ$ indicate the location of the source points in $\Omega^{e}$ and $\diamond$ those in $\Omega^{i}$. The collocation points on $\Gamma$ are identified by the markers ' $'$ '.

This section comprises three examples of increasing difficulty illustrating the efficiency of the MFS. The first shows the MFS performance for foams presenting geometrical singularities such as corners. In the second example, we tackle circular shaped corrugated foams. The last example concerns scatterers of arbitrary shapes. Because there are no analytical solutions to these problems, all reference solutions are computed using Finite Element models. These computations are carried using Lagrange quadratic finite elements in both fluid and poroelastic domains. The $\left(\mathbf{u}, p_{p}\right)$ formulation of Biot's equations $[5,11]$ is used and the non reflecting boundary conditions are implemented using Bermudez's perfectly matched layer (PML) formulation [7]. As for the accuracy of these FE reference solutions, numerical tests carried out on the circular shaped scattering problem shows that these results are reliable up to around 1 percent of error on the solid phase normal displacement which is acceptable if engineering accuracy is sought.

\subsection{Square scatterer}

The first example concerns that of a square-shaped poroelastic scatterer of side length $a=0.2 \mathrm{~m}$. The incident plane wave is horizontal, travelling in the $\zeta=(1,0)$ direction and the frequency is $1500 \mathrm{~Hz}$. In this scenario, the boundary curve is not regular and source points around the square are placed along the normal to the boundary avoiding the corners, see Fig. 3. In this respect, we found that putting more sources in the vicinity of the corners did not show any improvement. Comparisons between the MFS and the FE results are conveniently shown in Fig. 4 for the pressure (Fig. 4a) and the normal displacement (Fig. 4b). Here, the abscissae $\theta$ refers the usual polar coordinate of the collocation points on the boundary (the square is centered at the origin). One can note the associated global errors are in good agreements with the $a$ posteriori error estimator as shown in Table 2.

As expected, the discrepancies reach a maximum near the corners and this is particularly noticeable for the displacement curve. The pressure remains more stable even near the corners and is free of spurious oscillations. Fortunately these spurious oscillations, present on the normal displacement curve have a low radiation efficiency and their effect on the SCS is negligible.

We should point out that, the displacement and pressure magnitudes do not correspond to any realistic case as we took $A^{\text {inc }}=1$ in our calculations.

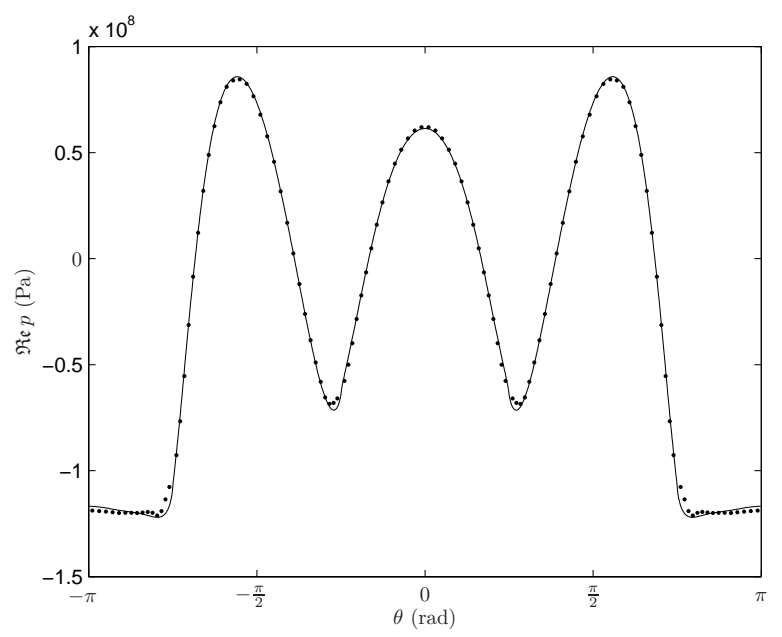

(a)

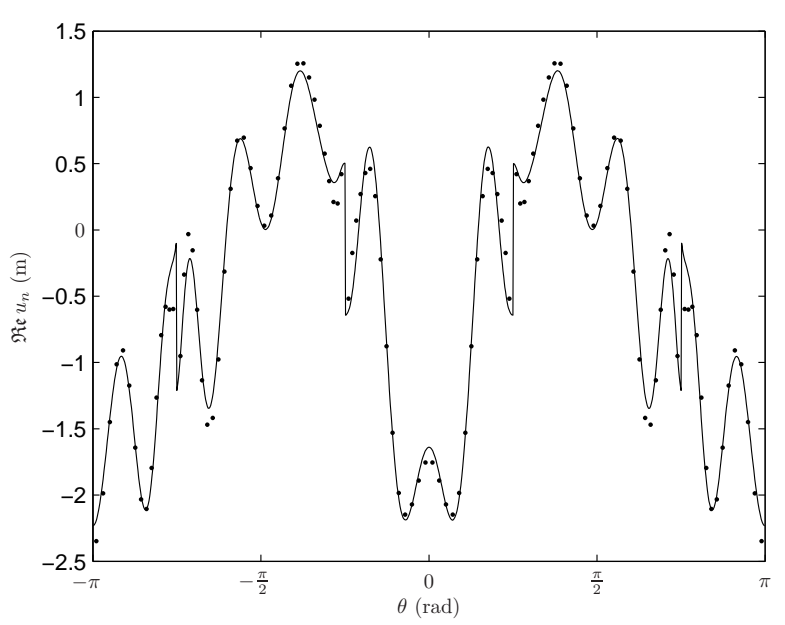

(b)

Figure 4: (a): Real part of the pressure field on $\Gamma$ at $1500 \mathrm{~Hz}$ for the square-shaped scatterer; solid line: FEM solution and dots refers to the MFS solution. (b): Real part of solid phase normal displacement.

\subsection{Scattering by a corrugated cylinder}

Here, we consider the scattering of a horizontal plane wave by a circular shaped corrugated foam with boundary 
Table 2: Relative errors on a square scatterer $(a=0.2 \mathrm{~m}, s=0.2 a)$ at $500 \mathrm{~Hz}$ and $1500 \mathrm{~Hz}$ for XFM foam with $Q=60$ and $2 \times 4 Q=480$ collocation points.

\begin{tabular}{|c|c|c|c|c|}
\hline & \multicolumn{2}{|c|}{$500 \mathrm{~Hz}$} & \multicolumn{2}{|c|}{$1500 \mathrm{~Hz}$} \\
\hline Fields & $\mathcal{E}_{\alpha}(\%)$ & $\hat{\mathcal{E}}(\%)$ & $\mathcal{E}_{\alpha}(\%)$ & $\hat{\mathcal{E}}(\%)$ \\
\hline$p(\alpha=\mathrm{a})$ & 5.56 & 0.75 & 3.95 & 1.03 \\
\hline $\mathbf{u} \cdot \mathbf{n}(\alpha=\mathrm{b})$ & 4.42 & 1.88 & 4.95 & 4.31 \\
\hline
\end{tabular}

given parametrically by

$$
\mathbf{x}(t)=0.1\left(\begin{array}{c}
\cos t \\
\sin t
\end{array}\right)+0.005\left(\begin{array}{c}
\cos 8 t \\
\sin 8 t
\end{array}\right), \quad t \in[0,2 \pi[.
$$

The shape of the boundary as well as the location of the collocation points and the source points are displayed in Fig. 5. The perfectly circular shape of radius $a=0.1 \mathrm{~m}$ is also illustrated. For this specific example, a multifrequency analysis is performed in the range $[0,5000 \mathrm{~Hz}]$. In Fig. 6 we show the evolution of the SCS for different materials: the XFM foam and the $\mathrm{B}$ wool whose properties are reported in Table 1 . The perfectly circular shape is also considered for the sake of illustration. The results are compared with the perfectly reflecting wall case (rigid wall). These are computed using the MFS with acoustic sources only and by applying the normal displacement condition at the interface: $\mathbf{w} \cdot \mathbf{n}=0$.

In the mid-frequency range, the presence of peaks for the XFM foam can be observed. An analysis of the poroelastic wave field in the absorbing material reveals that these are due to some resonance effects of the elastic foam's skeleton. For the wool, these peaks are highly attenuated and the use of an equivalent fluid model [12] would have been sufficient for the analysis. The effects of the corrugations on the SCS are noticeable above $3000 \mathrm{~Hz}$ for the rigid case. This frequency corresponds to an acoustic wavelength which is comparable to the length of the obstacle. For porous scatterers, the effects of the corrugations are dampened due to the absorbing properties of the materials, especially in the high-frequency range.

All the results have been computed with an accuracy that does not exceed $1.2 \%$ error. A closer analysis, however, is instructive as it shows behaviors which are inherent to the method. In Fig. 7, the associated a posteriori errors $\mathcal{E}_{\mathrm{b}}$ are plotted in logarithmic scale. For the rigid circular cylinder case, clearly identifiable peaks can be observed at the frequencies: 1626, 2592 and $3733 \mathrm{~Hz}$. These peaks correspond to a loss of accuracy due to some non-uniqueness problems of the MFS formulation. This point is discussed in length in [32] and in [10] for the circular cylinder case. It emerges that these 'critical' frequencies are in fact the eigenvalues of an interior problem corresponding to the internal surface on which are located the source points. Because the source points are placed on a perfectly circular ring, it was easy to check that the frequencies correspond to the interior Dirichlet problem [10]. In general,

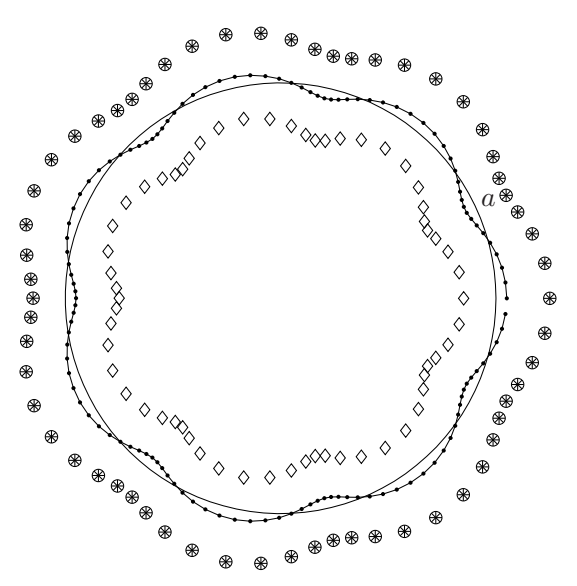

Figure 5: Corrugated cylinder shape. Here,$+ \times, \circ$ are the source points in $\Omega^{e}$ and $\diamond$ those in $\Omega^{i}$. The collocation points on $\Gamma$ are given by markers ' $'$ '.

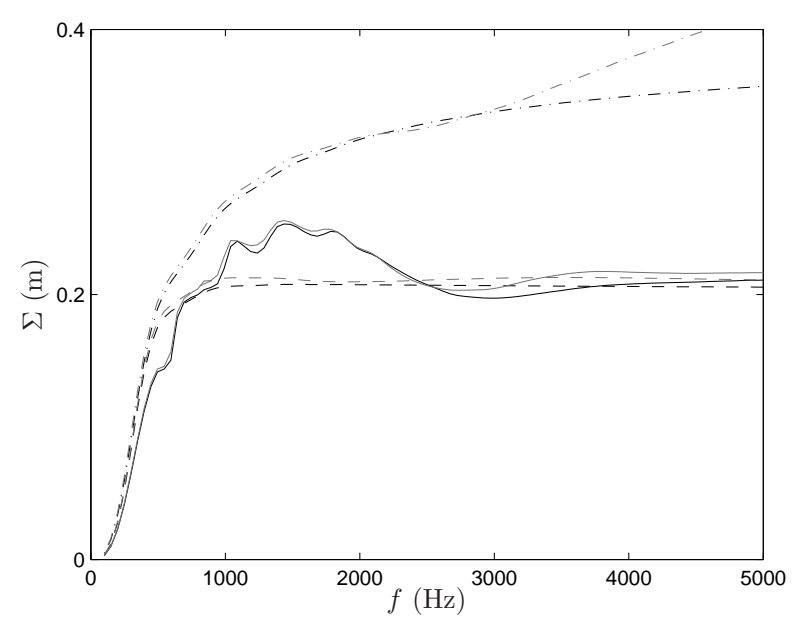

Figure 6: Scattering cross section for the straight (black) and corrugated cylinder (gray). - XFM foam, - - B wool, - - - rigid wall. Computed using $Q=60$.

these irregular frequencies are hard (if not impossible) to predict though their effects are somewhat noticeable even with porous scatterers (except with the B wool where it is harder to conclude).

In this work, the MFS algorithm was implemented using MATLAB and, for the specific examples illustrated here, around 0.73 second (CPU time) is needed per frequency (Intel Core 2 Duo T7100 @ 1.80 GHz, 2 GB RAM). To the authors' knowledge these performances cannot be met with the FEM. Using a compiled language and avoiding some redundant evaluations of Hankel functions, it is anticipated that the computational time could be reduced further by a factor of 10 . For the sake of illustration, the total pressure field is shown in Fig. 8. This corresponds to a high frequency calculation $(15000 \mathrm{~Hz})$. Around $1.6 \mathrm{sec}-$ onds are required to compute the solution with less than 


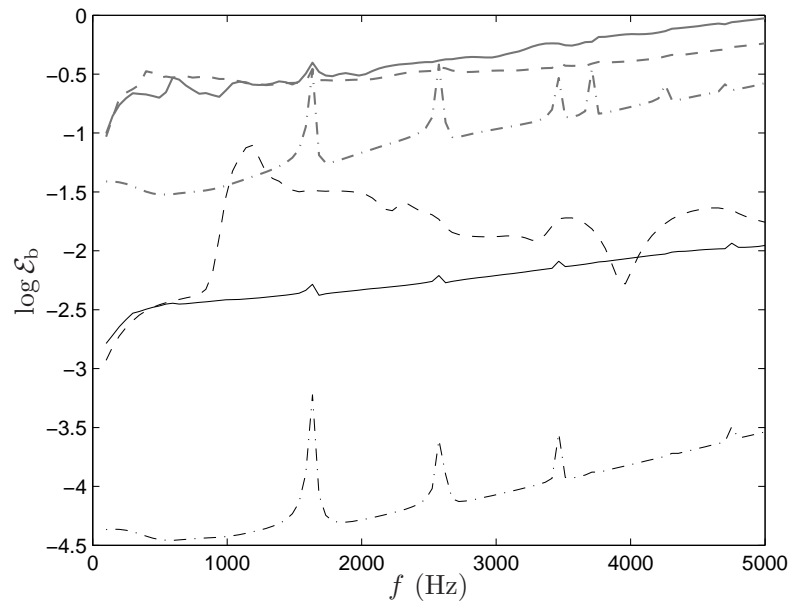

Figure 7: a posteriori error estimator for the straight (black) and corrugated (gray) cylinder. $\longrightarrow$ XFM foam, $--\mathrm{B}$ wool, - - - rigid wall. Computed using $Q=60$.

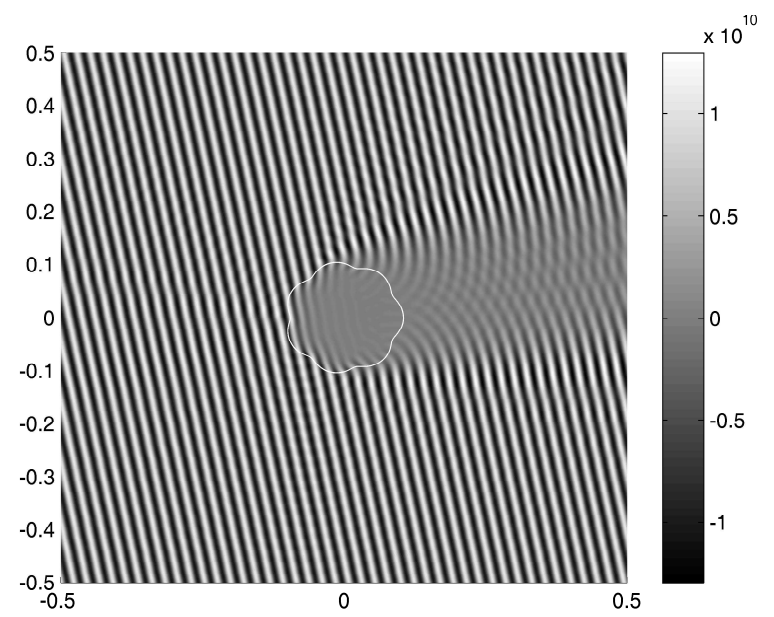

Figure 8: Real part of the pressure fields at $15000 \mathrm{~Hz}$ for a XFM foam obstacle impinged by plane wave with angle of incidence $\theta^{\text {inc }}=0.2$ rad.

$1.2 \%$ of a posteriori error and only 400 degrees of freedom (dof) are needed $(Q=100$ and we took $s=0.25 a$ for the sources locations). The computation of the pressure on a refined grid containing about 150,000 points takes approximately 30 seconds. Note the performance of the method could be further enhanced for this example and those of sec. 3 and 4.1 if symmetries of the domains were taken into account.

\subsection{Multiple scatterers of arbitrary shapes}

The last numerical experiment concerns a multiple scattering problem with non-convex objects having the shape of the three letters ' $\mathrm{M}$ ', ' $\mathrm{F}$ ' and 'S'. The boundaries have been drawn using a graphical software and interpolated with Bezier curves. Fig. 9 shows the locations of the source points as well as the collocation points on the letter ' $\mathrm{M}$ '.
The example considered here is that of an incident plane wave in the $\zeta=(\cos \pi / 4,-\sin \pi / 4)$ direction and the frequency is $6000 \mathrm{~Hz}$. In our calculation, we took $Q=80$ for each scatterer; this yields $N_{\text {dof }}=3 \times 4 \times Q=960$ variables. The a posteriori errors for the pressure and the displacement are $\mathcal{E}_{\mathrm{a}}=1.78 \%$ and $\mathcal{E}_{\mathrm{b}}=2.77 \%$. The total pressure in the acoustic domain and in the porous domain is shown in Fig. 10. The absorbing properties of the porous material can be visually identified. Note that the CPU time needed for computing the matrix coefficients and inverting the system does not exceed $20 \mathrm{~s}$ on a simple PC. The use of the FEM would be far more demanding in order to achieve the same level of accuracy.

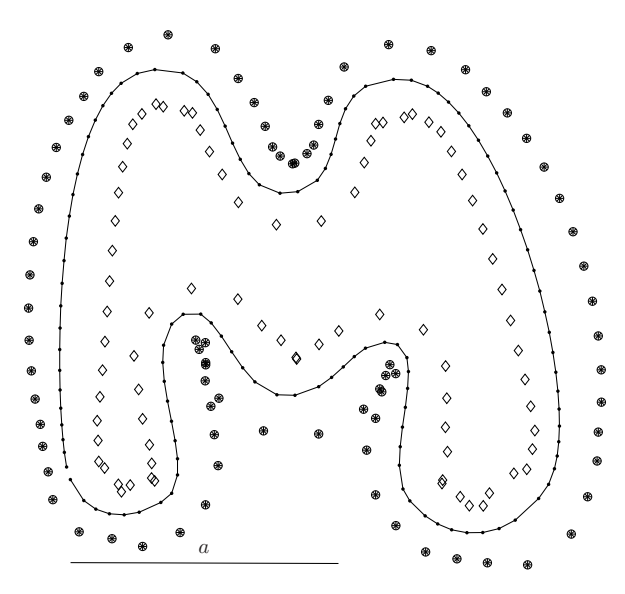

Figure 9: M-shaped scatterer with $a=0.1 \mathrm{~m}$. Here the symbols + , $\times$, $\circ$ indicate the location of the source points in $\Omega^{e}$ and $\diamond$ those in $\Omega^{i}$. The collocation points on $\Gamma$ are identified by the markers '.' .

\section{Periodic scattering}

Let us now consider an infinite periodic array of identical porous structures. The problem is depicted in Fig. 11. Here the geometry is assumed $d$-periodic in the $\mathbf{e}_{1}$ direction. When the array is illuminated by a plane wave of angle of incidence $\theta^{\text {inc }}$ (defined about the vertical axis as shown), the solution of the scattering problem is pseudoperiodic, that is $d$-periodic with a phase shift, and each physical variable (call it $X$ ) must fulfill the condition

$$
X\left(\mathbf{x}+m d \mathbf{e}_{1}\right)=X(\mathbf{x}) \mathrm{e}^{\mathrm{i} m \beta d} .
$$

Here $m$ is a relative integer and $\beta=k_{0} \sin \theta^{\text {inc }}$ is the projection of the incident wave-number vector on the $\mathbf{e}_{1}$ direction. This problem can be easily solved with the MFS by simply modifying the Green's function for the acoustic domain. The simplest form is a representation as a sum of images

$$
G_{\beta}^{d}(\mathbf{x}, \mathbf{y})=-\frac{\mathrm{i}}{4} \sum_{m=-\infty}^{\infty} H_{0}\left(k_{0} r_{m}\right) \mathrm{e}^{\mathrm{i} m \beta d}
$$




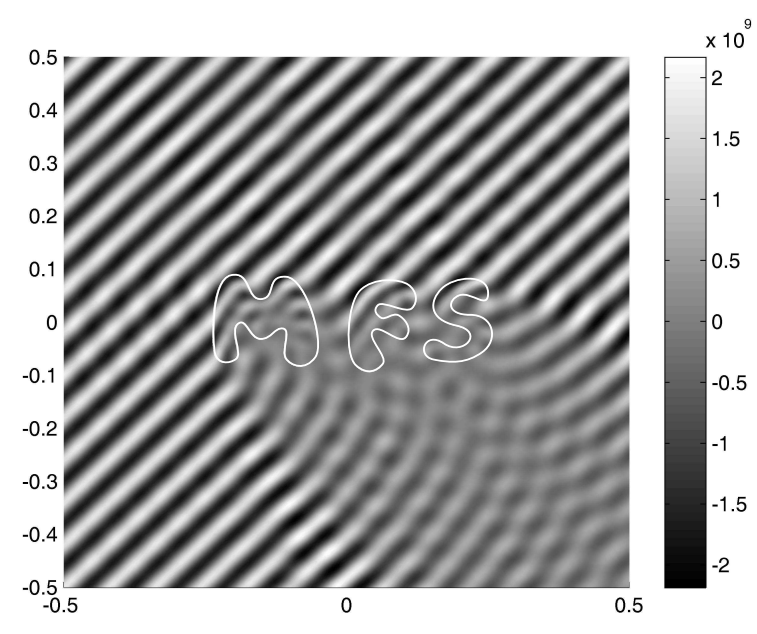

Figure 10: Real part of the total pressure in the acoustic domain and in the porous material (XFM foam).

For convenience we shall write the field point $\mathbf{x}=\left(x_{1}, x_{2}\right)$, the source point $\mathbf{y}=\left(y_{1}, y_{2}\right)$ and introduce the notation $\delta_{1}=x_{1}-y_{1}$ and $\delta_{2}=x_{2}-y_{2}$. In (25), $r_{m}=$ $\sqrt{\delta_{2}^{2}+\left(\delta_{1}-m d\right)^{2}}$ is the distance between the field point and the source point of the $m^{\text {th }}$ scatterer. The series is unfortunately very slowly convergent and is therefore not appropriate for numerical computations. In [20], the author has compared several methods to efficiently compute the $2 \mathrm{D}$ periodic Green's function $G_{\beta}^{d}$. Through extensive numerical tests it was shown that the Ewald method is probably the best technique giving a good trade-off between CPU time and robustness. This is based on the alternative form for Green's function:

$$
\begin{aligned}
G_{\beta}^{d}(\mathbf{x}, \mathbf{y}) & =\frac{1}{4 d} \sum_{m=-\infty}^{\infty} \frac{\mathrm{e}^{\mathrm{i} \beta_{m} \delta_{1}}}{\gamma_{m}}\left[\mathrm{e}^{\gamma_{m} \delta_{2}} \operatorname{erfc}\left(\frac{\gamma_{m} d}{2 e}+\frac{e \delta_{2}}{d}\right)\right. \\
& \left.+\mathrm{e}^{-\gamma_{m} \delta_{2}} \operatorname{erfc}\left(\frac{\gamma_{m} d}{2 e}-\frac{e \delta_{2}}{d}\right)\right] \\
& +\frac{1}{4 \pi} \sum_{m=-\infty}^{\infty} \mathrm{e}^{\mathrm{i} m \beta d} \sum_{n=0}^{\infty} \frac{1}{n !}\left(\frac{k_{0} d}{2 e}\right)^{2 n} E_{n+1}\left(\frac{e^{2} r_{m}^{2}}{d^{2}}\right),
\end{aligned}
$$

Here, $\beta_{m}=\beta+2 \pi m / d$ and

$$
\gamma_{m}=\left(\beta_{m}^{2}-k_{0}^{2}\right)^{\frac{1}{2}}=-\mathrm{i}\left(k_{0}^{2}-\beta_{m}^{2}\right)^{\frac{1}{2}} .
$$

The definition for the square root is chosen in order to ensure that the scattered acoustic waves are radiating away from the array or decay exponentially. The advantage of such a representation is that the series is quickly convergent and only a small number of terms is needed to achieve extremely accurate results (to double standard precision if necessary). Furthermore, the complementary Error function erfc and the exponential integral

$$
E_{n}(x)=\int_{1}^{\infty} u^{-n} \mathrm{e}^{-x u} \mathrm{~d} u
$$

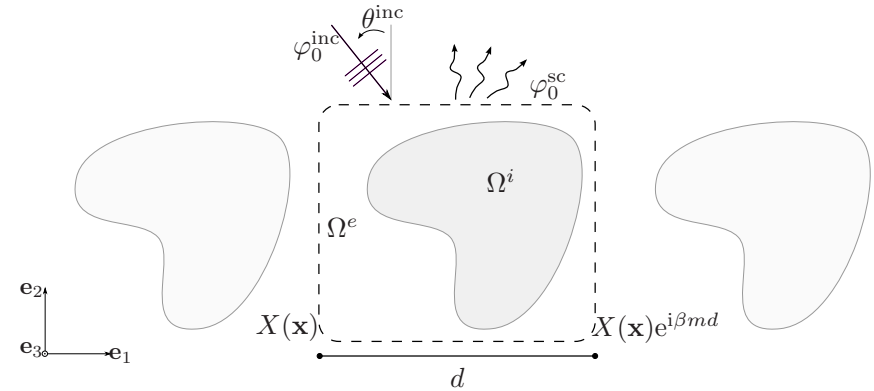

Figure 11: Periodic scattering geometry.

can be computed quickly [33]. In (26), the positive constant $e$ is arbitrary though its value will affect the convergence of the two summations, this is discussed in [20] and references therein. As shown in Table 3 in [20], $e=4$ seems to be a good choice allowing fast convergence and it was observed that less than twenty terms are required in the two summations over $m$ and around ten in the summation over $n$ to nearly achieve machine precision. The spatial derivatives involved in the computation of the displacement vector and the stress tensor are obtained by simply differentiating the truncated version of (26).

As an example, we consider an infinite periodic array of 'M'-shaped obstacles filled with XFM foam (Fig. 9). The results for the pressure field around the scatterer located near the origin $(m=0)$ are plotted in polar coordinates in Fig. 12. In our calculations, we took $f=1500 \mathrm{~Hz}, d=0.3$ $\mathrm{m}, \theta^{\text {inc }}=\pi / 6, Q=80$ and the location for the source points is chosen with $s=0.2 a$ ( $a$ is the characteristic length of the obstacle as shown Fig. 9). The MFS results are compared with those computed using pseudo-periodic finite elements as described in [17]. The a posteriori error estimator is $\mathcal{E}_{\mathrm{b}}=2 \%$ which is in accordance with the $3 \%$ of relative error measured against the FEM solution. Once the MFS coefficients have been found, quantities such as the reflection and transmission coefficients or the acoustic absorption can be easily computed in a straight forward manner using the plane wave decomposition for the periodic Green's function and we refer to [21] for more details. The effects of the periodic array on the scattering can be identified in Fig. 13 where the real part of the total pressure is shown around and inside the porous scatterers.

\section{Conclusions}

The application of the MFS for the acoustic wave scattering by porous scatterers has been presented. The wave equations for the fluid phase and solid phase displacements can be decoupled using the Helmholtz decomposition which allows for a systematic and simple way to construct fundamental solutions describing the wave displacement field in the poroelastic material. An assessment of the MFS efficiency with respect to the number and position of the virtual sources and of the collocation points is presented in detail for the specific case of a circular- 


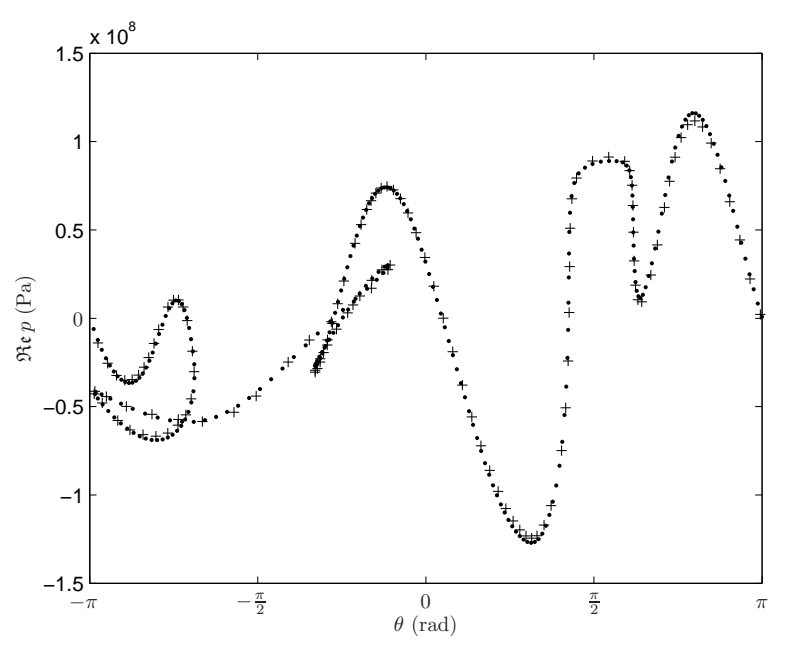

Figure 12: Real part of the pressure field around a M-shaped scatterer $(m=0)$ at $1500 \mathrm{~Hz}$. The marker '.' refers to the FEM solution and ' + ' to the MFS solution.

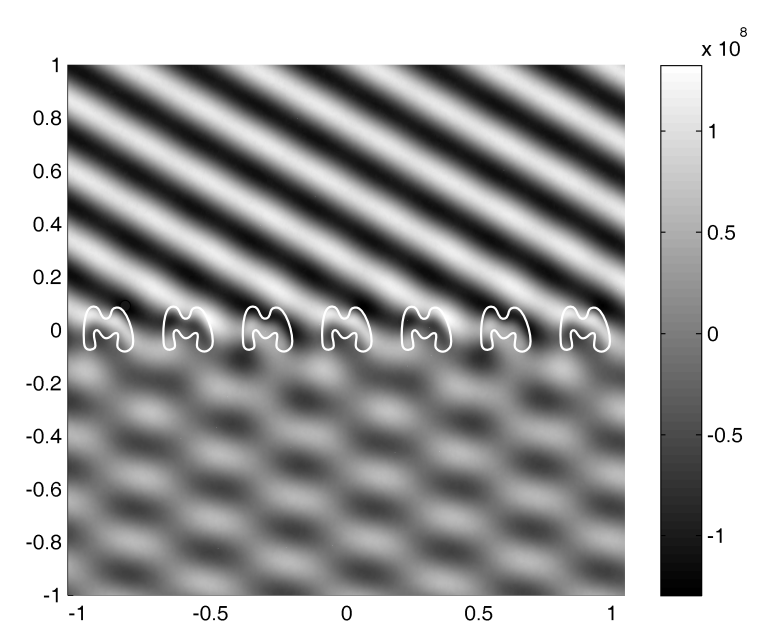

Figure 13: Real part of the MFS pressure field at $1500 \mathrm{~Hz}$ for a periodic 'M'-shaped porous scatterer.

shaped scatterer. In particular, it is shown that reliable error indicators can be used to assess the quality of the results. Through many illustrative examples of increasing difficulty, comparisons with results computed using a mixed pressure-displacement finite element formulation illustrate the considerable advantages of the MFS both in terms of computational resources and mesh preparation. Finally, the extension of the method to dealing with the acoustic wave scattering by an infinite array of periodic porous scatterers is discussed and presented. It is believed that the method show promise in solving a wide range of noise control problems ranging from multiple scattering to shape foam optimization. The development of the MFS for $3 \mathrm{D}$ problems is also of particular interest to us and this could be the subject of futur work.

\section{Acknowledgement}

The authors wish to thank the Projet Pluri-Formations PilcAm2 [28] at the Université de Technologie de Compiègne for providing the high performance computing resources that have contributed to the research results reported within this paper as well as the reviewers for their helpful comments.

\section{Appendix A. Scattering of a horizontal acoustic plane wave by a circular-shaped poroe- lastic scatterer.}

Take an incident plane wave along the $\mathbf{e}_{1}$ direction with amplitude $A^{\text {inc }}$. Following [1, 23], the outer wave field in the acoustic domain can be represented by separable solutions in polar coordinates $(r, \theta)$ as

$$
\begin{aligned}
\varphi_{0}^{\mathrm{inc}} & =A^{\mathrm{inc}} \mathrm{e}^{\mathrm{i} k_{0} x_{1}}=A^{\mathrm{inc}} \mathrm{e}^{\mathrm{i} k_{0} r \cos \theta} \\
& =A^{\mathrm{inc}}\left[J_{0}\left(k_{0} r\right)+2 \sum_{m=1}^{\infty} \mathrm{i}^{m} J_{m}\left(k_{0} r\right) \cos m \theta\right],
\end{aligned}
$$

and, similarly for the total field:

$$
\varphi_{0}=\varphi_{0}^{\mathrm{inc}}+\sum_{m=0}^{\infty} \hat{A}_{0, m} H_{m}\left(k_{0} r\right) \cos m \theta .
$$

For the wave field in the poroelastic medium we can follow a recent paper [24] and expand each potential as the Fourier series:

$$
\begin{aligned}
\varphi_{i} & =\sum_{m=0}^{\infty} \hat{A}_{i, m} J_{m}\left(k_{i} r\right) \cos m \theta, \text { with } i=1,2, \\
\varphi_{3} & =\sum_{m=0}^{\infty} \hat{A}_{3, m} J_{m}\left(k_{3} r\right) \sin m \theta .
\end{aligned}
$$

Now, applying the boundary conditions (5) at the airporous interface $r=a$ yields a linear system of the form

$$
\mathrm{MA} \hat{\mathrm{A}}_{m}=\mathrm{F}
$$

where all the quantities involved depend on the azimuthal mode number $m=0,1,2 \ldots$. The resolution of this $4 \times 4$ matrix system gives the coefficients $\hat{A}_{i, m}(i=0, \ldots, 3)$ and the analytical solution can thus be computed. For completeness, the analytical expressions for the coefficients are explicitly written:

$$
\begin{aligned}
& \mathrm{M}_{1,1}=\rho_{0} \omega^{2} H_{m}\left(k_{0} a\right) \\
& \mathrm{M}_{1,2}=-\frac{k_{1}^{2} J_{m}\left(k_{1} a\right)\left(Q+\mu_{1} R\right)}{\phi} \\
& \mathrm{M}_{1,3}=-\frac{k_{2}^{2} J_{m}\left(k_{2} a\right)\left(Q+\mu_{2} R\right)}{\phi} \\
& \mathrm{M}_{1,4}=0
\end{aligned}
$$




$$
\begin{aligned}
& \mathrm{M}_{2,1}=\frac{-k_{0} H_{m+1}\left(k_{0} a\right) a+m H_{m}\left(k_{0} a\right)}{a} \\
& \mathrm{M}_{2,2}=-\frac{\phi_{1}\left(-k_{1} J_{m+1}\left(k_{1} a\right) a+m J_{m}\left(k_{1} a\right)\right)}{a} \\
& \mathrm{M}_{2,3}=-\frac{\phi_{2}\left(-k_{2} J_{m+1}\left(k_{2} a\right) a+m J_{m}\left(k_{2} a\right)\right)}{a} \\
& \mathrm{M}_{2,4}=-\frac{J_{m}\left(k_{3} a\right) m \phi_{3}}{a}
\end{aligned}
$$$$
\mathrm{M}_{3,1}=-\rho_{0} \omega^{2} H_{m}\left(k_{0} a\right)
$$$$
\mathrm{M}_{3,2}=\frac{-2 N k_{1} J_{m+1}\left(k_{1} a\right) a+J_{m}\left(k_{1} a\right) C_{1}}{a^{2}}
$$$$
\mathrm{M}_{3,3}=\frac{-2 N k_{2} J_{m+1}\left(k_{2} a\right) a+J_{m}\left(k_{2} a\right) C_{2}}{a^{2}}
$$$$
\mathrm{M}_{3,4}=\frac{-2 m N}{a^{2}}\left(-k_{3} J_{m+1}\left(k_{3} a\right) a+J_{m}\left(k_{3} a\right)(m-1)\right)
$$

$$
\begin{aligned}
& \mathrm{M}_{4,1}=0 \\
& \mathrm{M}_{4,2}=\frac{2 m N}{a^{2}}\left(-k_{1} J_{m+1}\left(k_{1} a\right) a+J_{m}\left(k_{1} a\right)(m-1)\right)
\end{aligned}
$$

$$
\mathrm{M}_{4,3}=\frac{2 m N}{a^{2}}\left(-k_{2} J_{m+1}\left(k_{2} a\right) a+J_{m}\left(k_{2} a\right)(m-1)\right)
$$

$$
\begin{aligned}
\mathrm{M}_{4,4}= & -\frac{N}{a^{2}}\left(-2 k_{3} J_{m+1}\left(k_{3} a\right) a\right. \\
& \left.+J_{m}\left(k_{3} a\right)\left(-2 m^{2}+2 m+k_{3}^{2} a^{2}\right)\right),
\end{aligned}
$$

with the constants

$$
C_{i}=k_{i}^{2}\left((Q+R) \mu_{i}+P+Q\right) a^{2}-2 m N(m-1),
$$

and $\phi_{i}=\left(\phi \mu_{i}+1-\phi\right)$. The right hand side vector contains the contributions from the incident plane wave:

$$
\begin{aligned}
& \mathrm{F}_{1}=-2 \rho_{0} \omega^{2} A^{\mathrm{inc}_{\mathrm{i}}^{m}} J_{m}\left(k_{0} a\right) \\
& \mathrm{F}_{2}=\frac{2}{a} A^{\mathrm{inc}} \mathrm{i}^{m}\left(k_{0} J_{m+1}\left(k_{0} a\right) a-m J_{m}\left(k_{0} a\right)\right) \\
& \mathrm{F}_{3}=2 \rho_{0} \omega^{2} A^{\mathrm{inc}} \dot{\mathrm{i}}^{m} J_{m}\left(k_{0} a\right) \\
& \mathrm{F}_{4}=0 .
\end{aligned}
$$

and these coefficients have to be divided by 2 for the special case $m=0$. The conditioning of the system can be improved by multiplying the line of $\mathrm{M}$ corresponding to the normal displacement condition by the factor $\rho_{0} c_{0} \omega$ (and similarly for the corresponding line of F). In practice, the summation of the series is carried out until the relative difference between two consecutive steps is lower than $10^{-10}$ percent.

\section{References}

[1] A. Abramowitz, I. Stegum, Handbook of Mathematical functions, New York, Dover Publ, 1965.
[2] J.F. Allard, Propagation of sound in porous media: modeling sound absorbing materials, Chapman \& Hall, 1993.

[3] C.J.S. Alves, On the choice of source points in the method of fundamental solutions, Engng. Anal. Bound. Elem 33 (2009) 1348-1361.

[4] C.J.S. Alves, S.S. Valtchev, Numerical comparison of two meshfree methods for acoustic wave scattering, Engng. Anal. Bound. Elem 29 (2005) 371-382.

[5] N. Atalla, M.A. Hamdi, R. Panneton, Enhanced weak integral formulation for the mixed $(u, p)$ poroelastic equations, J. Acoust. Soc. Am. 109 (2001) 3065-3068.

[6] N. Atalla, R. Panneton, F.C. Sgard, X. Olny, Acoustic absorption of macro-perforated porous materials, J. Sound Vib. 243 (2001) 659-678.

[7] A. Bermudez, L. Hervella-Nieto, A. Prieto, R. Rodriguez, An optimal perfectly matched layer with unbounded absorbing function for time-harmonic acoustic scattering problems, J. Comput. Phy. 223 (2007) 469-488.

[8] M.A. Biot, Theory of propagation of elastic waves in a fluidsaturated porous solid. I. low-frequency range., J. Acoust. Soc. Am. 28 (1956) 168-191.

[9] G. Bonnet, Basic singular solutions for a poroelastic medium in the dynamic range, J. Acoust. Soc. Am. 82 (1987) 1758-1762.

[10] I.L. Chen, Using the method of fundamental solutions in conjunction with the degenerate kernel in cylindrical acoustic problems, J. Chin. Inst. Engin. 29 (2006) 445-457.

[11] P. Debergue, R. Panneton, N. Atalla, Boundary conditions for the weak formulation of the mixed $(u, p)$ poroelasticity problem, J. Acoust. Soc. Am. 106 (1999) 2393-2390.

[12] O. Doutres, N. Dauchez, J.M. Génevaux, O. Dazel, Validity of the limp model for porous materials: a criterion based on the Biot theory, J. Acoust. Soc. Am. 122 (2007) 2038-2048.

[13] G. Fairweather, A. Karageorghis, The method of fundamental solutions for elliptic boundary value problems, Adv. Comput. Math. 9 (1998) 69-95.

[14] G. Fairweather, A. Karageorghis, P.A. Martin, The method of fundamental solutions for scattering and radiation problems, Engng. Anal. Bound. Elem. 27 (2003) 759-769.

[15] S.M. Hasheminejad, S.A. Badsar, Acoustic scattering by a pair of poroelastic spheres, J. Mech. Appl.Math. 46 (2004) 95-113.

[16] S.M. Hasheminejad, R. Sanaei, Acoustic scattering by an elliptic cylindrical absorber, Acta Acustica united with Afcustica 93 (2007) 789-803.

[17] A.C. Hennion, R. Bossut, J.N. Decarpigny, C. Audoly, Analysis of the scattering of a plane acoustic wave by a periodic elastic structure using the finite element method : application to compliant tube gratings, J. Acoust. Soc. Am. 87 (1990) 1861-1870.

[18] N.E. Horlin, M. Nordstron, P. Goransson, A 3-D hierarchical fe formulation of Biot's equations for elasto-acoustic modeling of porous media, J. Sound Vib. 245 (2001) 633-652.

[19] J.S. Lee, Y.Y. Kim, J.S. Kim, Y.J. Kang, Two-dimensional poroelastic acoustical foam shape design for absorption coefficient maximization by topology optimization method, J. Acoust. Soc. Am. 123 (2008) 2094-2106.

[20] C.M. Linton, The Green's function for the two dimensional Helmholtz equation in periodic domains, J. Engin. Math. 33 (1998) 377-402.

[21] C.M. Linton, I. Thompson, Resonant effects in scattering by periodic arrays, Wave motion 44 (2007) 167-175.

[22] O.L. Lovera, Boundary conditions for a fluid-saturated porous solid, Geophysics 52 (1987) 174-178.

[23] P.M. Morse, K.U. Ingard, Theoretical acoustics, Princeton University Press, 1986.

[24] B. Nennig, E. Perrey-Debain, M. Ben Tahar, A mode matching method for modelling dissipative silencers lined with poroelastic materials and containing mean flow, J. Acoust. Soc. Am. 128 (2010) 3308-3320.

[25] B. Nennig, E. Perrey-Debain, J.D. Chazot, On the efficiency of the method of fundamental solutions for acoustic scattering by a poroelastic material, in: The 32nd International Conference on Boundary Elements and other Mesh Reduction Methods 
(BEM/MRM), New Forest, UK, pp. 181-193.

[26] X. Olny, C. Boutin, Acoustic wave propagation in double porosity media, J. Acoust. Soc. Am. 114 (2005) 73-89.

[27] E. Perrey-Debain, Plane wave decomposition in the unit disc: Convergence estimates and computational aspects, J. Comput. Appl. Math. 193 (2006) 140-156.

[28] Pilcam, http://pilcam2.wikispaces.com, Last visit october 2010.

[29] S. Rigobert, N. Atalla, F. Sgard, Investigation of the convergence of the mixed displacement-pressure formulation for threedimensional poroelastic materials using hierarchical elements, J. Acoust. Soc. Am. 114 (2003) 2607-2617.

[30] F.C. Sgard, X. Olny, N. Atalla, F. Castel, On the use of perforations to improve the sound absorption of porous materials, Appl. Acoust. 66 (2005) 625-651.

[31] O. Tanneau, P. Lamary, Y. Chevalier, A boundary element method for porous media, J. Acoust. Soc. Am. 20 (2006) 12391251.

[32] D.T. Wilton, I.C. Mathews, R.A. Jeans, A clarification of nonexistence problems with the superposition method, J. Acoust. Soc. Am. 94 (1993) 1676-1680.

[33] S. Zhang, J. Jin, Computation of Special Functions, Wiley, 1996. 